\title{
OPEN Emulating the local Kuramoto model with an injection-locked photonic crystal laser array
}

\author{
Naotomo Takemura ${ }^{1,2}$, Kenta Takata ${ }^{1,2}$, Masato Takiguchi ${ }^{1,2}$ \& Masaya Notomi ${ }^{1,2} \bowtie$
}

The Kuramoto model is a mathematical model for describing the collective synchronization phenomena of coupled oscillators. We theoretically demonstrate that an array of coupled photonic crystal lasers emulates the Kuramoto model with non-delayed nearest-neighbor coupling (the local Kuramoto model). Our novel strategy employs indirect coupling between lasers via additional cold cavities. By installing cold cavities between laser cavities, we avoid the strong coupling of lasers and realize ideal mutual injection-locking with effective non-delayed dissipative coupling. First, after discussing the limit cycle interpretation of laser oscillation, we demonstrate the synchronization of two indirectly coupled lasers by numerically simulating coupled-mode equations. Second, by performing a phase reduction analysis, we show that laser dynamics in the proposed device can be mapped to the local Kuramoto model. Finally, we briefly demonstrate that a chain of indirectly coupled photonic crystal lasers actually emulates the one-dimensional local Kuramoto chain. We also argue that our proposed structure, which consists of periodically aligned cold cavities and laser cavities, will best be realized by using state-of-the-art buried multiple quantum well photonic crystals.

Nowadays, the investigation of synergetic dynamics emerging from coupled oscillators is an interdisciplinary study intensively discussed in physics, mathematics, chemistry, biology, and neuroscience ${ }^{1}$. Collective phenomena in coupled oscillators were investigated for the first time by Kuramoto, who used a large set of fully-connected oscillators which is a mathematical model called the Kuramoto model ${ }^{2,3}$. In spite of the simplicity of the Kuramoto model, it comprises rich physics. For example, it exhibits a phase transition-like phenomenon from an incoherent state to a fully synchronized state when coupling strength reaches a threshold. Investigations of the Kuramoto model are not limited to theoretical ones. Actually, it is the simplest model for understanding various collective synchronization phenomena observed in nature, such as the collective synchronizations of neural oscillations and fireflies. Another actively studied direction is the emulation of the Kuramoto model with physical systems, for which the well-known example is the Josephson junction array ${ }^{4-6}$, though another promising approach is to use an array of coupled lasers ${ }^{7-17}$. In this paper, we employ the latter approach and focus on a nanophotonic device, which provides an attractive playground for studying dynamical systems, with which synchronization of limit cycle oscillations has been theoretically and experimentally investigated ${ }^{18-23}$.

We propose a novel nanophotonic device that emulates the Kuramoto model with non-delayed nearestneighbor coupling ${ }^{3,24-28}$, which we call the local Kuramoto model. Our idea is inspired by pioneering studies on coupled photonic crystal (PhC) lasers ${ }^{29-34}$ and by the mutual injection locking technique in laser physics ${ }^{16,35-40}$. Different from the conventional injection-locking ${ }^{41,42}$, mutual injection-locking involves neither master nor slave lasers. Our proposed device employs PhC lasers indirectly coupled via additional cold cavities. We demonstrate that the cold cavities play a crucial role in avoiding strong coupling between lasers, which results in ideal mutual injection-locking and dramatically simplifies the phase dynamics of laser oscillations. Compared with the other systems, nanophotonic Kuramoto models can be very compact devices that operate even at room temperatures. Furthermore, using PhC lasers, we aim for an on-chip realization of the local Kuramoto model, which may have an application as a coherent high-power laser. Additionally, in contrast to delayed coupling due to optical paths in free-space injection-locking ${ }^{11,16}$, our on-chip local Kuramoto model can provide stable coupling without coupling delay thanks to the direct evanescent coupling. Actually, the realization of dissipative coupling without time delay will be very difficult without using our scheme.

First, as a starting point, we consider two coupled $\mathrm{PhC}$ lasers coupled via a cold cavity. For this purpose, we interpret laser oscillation as limit cycle oscillation and model it by the Stuart-Landau equation. With

\footnotetext{
${ }^{1}$ Nanophotonics Center, NTT Corp., 3-1, Morinosato Wakamiya Atsugi, Kanagawa 243-0198, Japan. ${ }^{2}$ NTT Basic Research Laboratories, NTT Corp., 3-1, Morinosato Wakamiya Atsugi, Kanagawa 243-0198, Japan. ${ }^{\square}$ email: masaya.notomi.mn@hco.ntt.co.jp
} 
coupled-mode equations, we numerically demonstrate the synchronization (mutual injection-locking) of two lasers. Furthermore, we confirm that strong-coupling between the two lasers is actually prohibited by the presence of the additional cold cavity. Second, in the same way as in our previous paper $^{43}$, we perform a phase reduction analysis to calculate the phase equations of motion for two indirectly coupled lasers ${ }^{2,44,45}$. The obtained phase equations of motion indicate that the phase dynamics of lasers indirectly coupled via cold cavities is equivalent to the local Kuramoto model. Finally, we demonstrate that a one-dimensional chain of indirectly coupled $\mathrm{PhC}$ lasers can emulate the one-dimensional local Kuramoto chain ${ }^{46}$.

We also argue that our proposed device can be realized best by using buried multiple quantum well (MQW) $\mathrm{PhC}$ cavities ${ }^{47-50}$, where MQWs are locally embedded in a PhC slab. With this state-of-the-art technology, laser and cold cavities can be periodically aligned on a PhC chip.

\section{Lasers as limit cycle oscillators}

Here, we review limit cycle interpretation for laser oscillation. In general, using complex field $\alpha$ and carrier number $N$, single-mode laser dynamics are, in the nonrotating frame, described by the following rate equations: ${ }^{51-53}$

$$
\begin{gathered}
\dot{\alpha}=-i \omega_{c} \alpha-\frac{1}{2} \gamma_{c} \alpha+\frac{1}{2} \beta \gamma_{\|} N \alpha \\
\dot{N}=-\gamma_{\|} N-\beta \gamma_{\|} N|\alpha|^{2}+P,
\end{gathered}
$$

where $P$ is the pumping rate for carriers, and $\omega_{c}$ is the resonance frequency of the laser cavity. Decay rates $\gamma_{c}$ and $\gamma_{\|}$are photon and carrier decay rates, respectively. Note that, in this paper, by employing the quantum optics convention, the electric field rotates as $\alpha(t)=\alpha(0) e^{-i \omega_{c} t}$, which is opposite to the rotation in conventional coupled-mode equations, $\left[\alpha(t)=\alpha(0) e^{i \omega_{c} t}\right]$. The coefficient $\beta$ represents the fraction of photons spontaneously emitted into a lasing mode, and it is called the spontaneous emission coupling coefficient $t^{51}$. For simplicity, we neglect the linewidth enhancement factor in the rate equations (1) and (2) in the main text. In Section 5 in the supplemental material, we discuss the effect of the linewidth enhancement factor on synchronization, which may be negligible in quantum-dot lasers but generally has non-negligible effects in semiconductor lasers. Here, it is worth noting that, in Eqs. (1) and (2), the terms $\frac{1}{2} \beta \gamma_{\|} N \alpha$ and $-\beta \gamma_{\|} N|\alpha|^{2}$ represent the stimulated emission, while there are no spontaneous emission terms. The effect of spontaneous emission will be included in the rate equations through a field noise term, if necessary. It is also important to note that Eqs. (1) and (2) hold only for a low $\beta(\ll 1)$, which is usually the case in most lasers. The rate equations (1) and (2) are known to exhibit Hopf bifurcation, which is equivalent to lasing, when the pump rate reaches a lasing threshold $P=P_{\text {th }}=\gamma_{c} / \beta$.

In this paper, for further simplification, we consider the case where the photon lifetime is much longer than the carrier lifetime $\left(\gamma_{c} \ll \gamma_{\|}\right)$, which is called the class-A condition ${ }^{54}$. With this assumption, we adiabatically eliminate the carrier degree of freedom as $\dot{N}=0^{55,56}$. The adiabatic elimination of the carrier dynamics reduces the rate equations (1) and (2) to

$$
\dot{\alpha}=-i \omega_{c} \alpha+\frac{1}{2} \gamma_{c} \varepsilon \alpha-\frac{1}{2} \beta \gamma_{c}|\alpha|^{2} \alpha
$$

Equation (3) is the well-known Stuart-Landau equation ${ }^{2,57}$, which is also called the Van der Pol equation ${ }^{21,58}$. Importantly, parameter $\varepsilon$ in Eq. (3) is the pump parameter defined as

$$
\varepsilon \equiv \frac{P-P_{\text {th }}}{P_{\text {th }}} \text { with } P_{\text {th }}=\frac{\gamma_{c}}{\beta},
$$

which indicates that the Hopf bifurcation (lasing) again occurs when $\varepsilon$ exceeds zero. Actually, when $\varepsilon>0$, the field amplitude $|\alpha|$ [see Fig. 1a] increases with an increase in the pump parameter as

$$
|\alpha|=\sqrt{\frac{\varepsilon}{\beta}} \text { for } \varepsilon \geq 0 .
$$

Therefore, in Eq. (3), the linear $\gamma_{c} \varepsilon \alpha / 2$ and nonlinear term $\beta \gamma_{c}|\alpha|^{2} \alpha / 2$ can be interpreted as gain and gain saturation, respectively. Here, it is important to stress that the laser oscillation itself is interpreted as limit cycle oscillation, and thus the resonance frequency of the laser cavity $\omega_{c}$ is the oscillation frequency of the limit cycle. As limit cycle oscillation emerges only in a nonlinear dissipative system with energy injection, lasing is achieved with the cavity decay, pumping, and gain saturation (nonlinearity).

Finally, we briefly comment on the effect of photon-carrier dynamics on synchronization properties, which will be important in real $\mathrm{PhC}$ cavity lasers. Since $\mathrm{PhC}$ cavity lasers are semiconductor lasers, their carrier lifetime is much longer than the photon lifetime (sometimes called class-B lasers ${ }^{54}$ ), and the relaxation oscillation appears around lasing threshold ${ }^{59,60}$. Therefore, in real $\mathrm{PhC}$ cavity lasers, the adiabatic elimination approximation of the carrier degree of freedom cannot be justified, and we need to directly simulate the rate equations (1) and (2). Fortunately, we found that Eqs. (1) and (2) quantitatively provide the same results as the Stuart-Landau equation as long as phase dynamics are concerned, which can also be confirmed with the phase equation of motion for the class-B rate equations. See Section 4 in the supplemental material. 
(a)

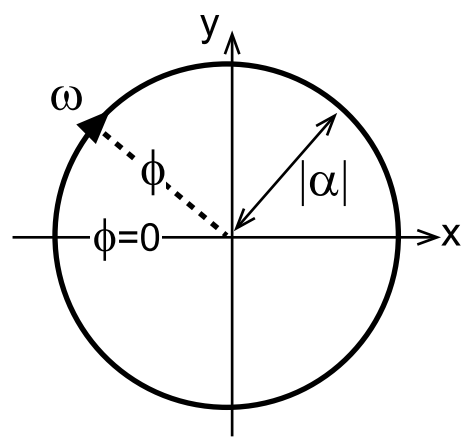

(b)

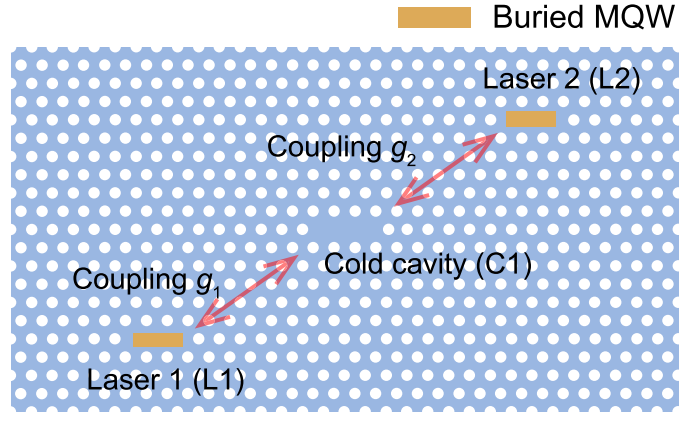

Figure 1. (a) Laser oscillation is interpreted as limit cycle oscillation in the nonrotating frame, where the laser frequency $\omega$ corresponds to the frequency of the limit cycle. With the amplitude $|\alpha|=\sqrt{\varepsilon / \beta}$ and phase $\phi=\omega t$ of the laser, we define the limit cycle orbit as $(x(\phi), y((\phi)))=\sqrt{\varepsilon / \beta}(-\cos \phi$, $\sin \phi)$, where $x$ and $y$ are the real $\operatorname{Re}[\alpha]$ and imaginary parts $\operatorname{Im}[\alpha]$ of the field, respectively. (b) Illustration of two PhC lasers (L1 and L2) indirectly coupled via a cold cavity (C1). The three cavities are evanescently coupled with coupling strengths $g_{1}$ and $g_{2}$.

\section{Synchronization of two lasers}

Coupled-mode equations. Now, we consider the device shown in Fig. 1b, where the two lasers (L1 and $\mathrm{L} 2)$ are indirectly coupled via the cold cavity (C1). The corresponding coupled-mode equations of motion representing field dynamics are given by

$$
\begin{gathered}
\dot{\alpha}_{1}=-i \omega_{1} \alpha_{1}+\frac{1}{2} \gamma_{1} \varepsilon_{1} \alpha_{1}-\frac{1}{2} \beta_{1} \gamma_{1}\left|\alpha_{1}\right|^{2} \alpha_{1}-i g_{1} E_{1} \\
\dot{E}_{1}=-i \Omega_{1} E_{1}-\frac{1}{2} \Gamma_{1} E_{1}-i g_{1} \alpha_{1}-i g_{2} \alpha_{2} \\
\dot{\alpha}_{2}=-i \omega_{2} \alpha_{2}+\frac{1}{2} \gamma_{2} \varepsilon_{2} \alpha_{2}-\frac{1}{2} \beta_{2} \gamma_{2}\left|\alpha_{2}\right|^{2} \alpha_{2}-i g_{2} E_{1},
\end{gathered}
$$

where $\alpha_{1,2}$ and $E_{1}$ represent fields in the laser cavity and cold cavity, respectively. Additionally, $\omega_{1,2}$ and $\Omega_{1}$ respectively represent the resonance frequencies of the laser cavities (L1 and L2) and coldcavity (C1). Similarly, $\gamma_{1,2}$ and $\Gamma_{1}$ are the field decay rates of the laser- $(\mathrm{L} 1,2)$ and cold cavity $(\mathrm{C} 1)$, respectively. The parameter $\beta_{1,2}$ is the spontaneous emission coupling coefficient, while $\varepsilon_{1,2}$ is the pump parameter for laser L1 and L2. Finally, the two coupling strengths between the cavities are denoted by $g_{1}$ and $g_{2}$. For simplicity, in the rest of this paper, we use $\beta_{1}=\beta_{2}=0.001$ and $\varepsilon_{1}=\varepsilon_{2}=1.0$, which is above the lasing threshold. Furthermore, we use the same values for the normalized decay rates of the laser cavity and cold cavity: $\Gamma_{1}=\gamma_{2}=\gamma_{1} \equiv 1$, where $\gamma_{1}$ is interpreted as a dimensionless parameter for numerical simulations.

To observe synchronization, we set the resonance frequencies of the two laser cavities as $\omega_{2}=\omega_{1}+\Delta \omega$ with $\Delta \omega=0.01 \gamma_{1}$, where $\Delta \omega \equiv \omega_{2}-\omega_{1}$ is the frequency difference between the two lasers. For the cold cavity $(\mathrm{C} 1)$, for simplicity, we use the same resonance frequency as $L 1: \Omega_{1}=\omega_{1}$.

Time evolutions. By showing field time evolutions described by the coupled-mode Eqs. (6)-(8), we demonstrate the synchronization of two lasers (mutual injection locking). Since the typical laser frequency, which is on the order of hundreds of terahertz, we perform the rotating-frame transformation for all fields, for example, as $\alpha_{1} e^{-i \omega_{s} t} \rightarrow \alpha_{1}$. With this rotating frame transformation, we shift the resonance frequencies of the cavities as $\omega_{1}^{\prime} \equiv \omega_{1}-\omega_{s}=1 \gamma_{1}, \omega_{2}^{\prime} \equiv \omega_{2}-\omega_{s}=1.01 \gamma_{1}$, and $\Omega_{1}^{\prime} \equiv \Omega_{1}-\omega_{s}=1 \gamma_{1}$. Importantly, there is an arbitrariness in the absolute frequencies, and only the relative frequencies are important. Thus, the frequency of the rotating frame, $\omega_{s}$, is arbitrary, and only the relative values between $\omega_{1}, \omega_{2}$, and $\Omega_{1}$ matter.

First, Fig. 2a shows the time evolutions of the real parts of the fields $\operatorname{Re}\left[\alpha_{1}(\mathrm{t})\right]$ (black) and $\operatorname{Re}\left[\alpha_{2}(\mathrm{t})\right]$ (blue) for the lasers L1 and L2, respectively, without coupling between cavities $g_{1}=g_{2}=0$. Without coupling between the cavities, there is no photon in cold-cavity $\mathrm{C} 3$, and thus $E_{1}(t)=0$. As we expect, in Fig. 2a, the fields in laser L1 and L2 oscillate with their own frequencies: $\omega_{1}^{\prime}=1 \gamma_{1}$ and $\omega_{2}^{\prime}=1.01 \gamma_{1}$. Second, we introduce coupling between the cavities as $g_{1}=g_{2}=0.1 \gamma_{1}$ in Fig. 2b, where the green curve is the time evolution of the real part of the field in cold-cavity $\mathrm{C} 1 \mathrm{Re}\left[\mathrm{E}_{1}\right]$. Figure $2 \mathrm{~b}$ indicates that the two indirectly coupled laser oscillations exhibit synchronization (mutual injection locking), which is the main result of this paper. Furthermore, the synchronization phase is anti-phase, which is called anti-phase synchronization. Importantly, thanks to cold-cavity C3, normal-mode splitting associated with strong coupling between the two lasers is prohibited, which is confirmed from the fact that the frequency of the synchronized oscillations does not depend on the initial states of two lasers (not shown). In fact, when the system is in the strong-coupling regime, depending on the initial states of two lasers, for example, they form a "bonding" or "anti-bonding" mode, and their frequencies become lower or higher than the original oscillation frequencies ${ }^{43}$. Importantly, no matter how weak the coupling is, directly coupled 
(a) Without coupling $\left(g_{12}=0\right)$

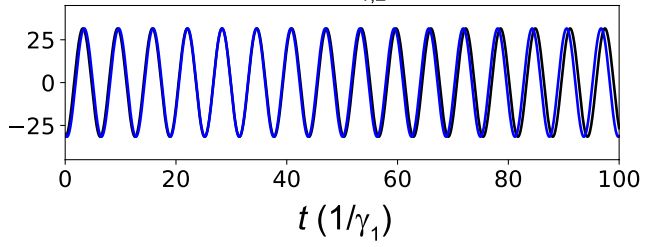

(c)

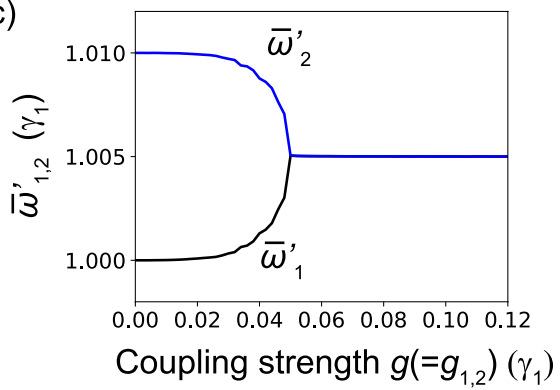

(b) With coupling $\left(g_{12}=0.1 \gamma_{1}\right)$

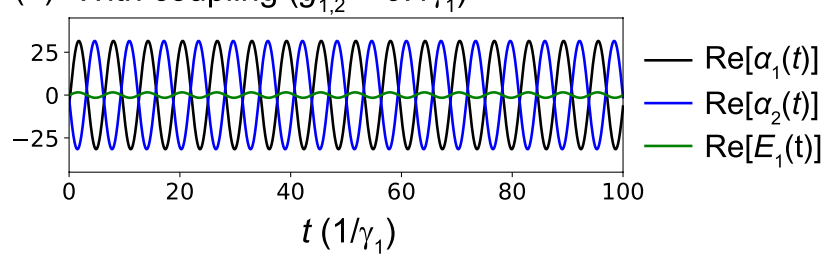

(d)

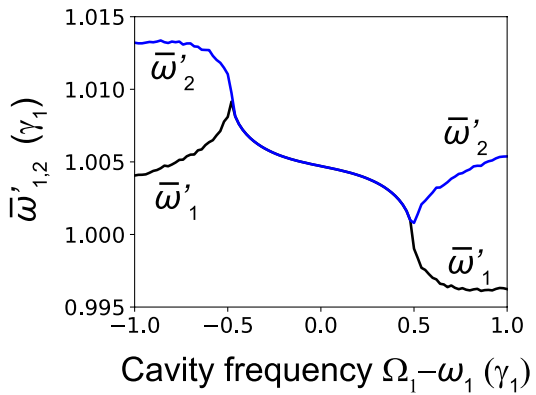

Figure 2. Simulations for two lasers [see Fig. 1b] coupled via a cold cavity. The simulated time evolutions of the real part of the field $\operatorname{Re}\left[\alpha_{1,2}(\mathrm{t})\right]$ without $g_{1,2}=0(\mathbf{a})$ and with coupling $g_{1,2}=0.1 \gamma_{1}(\mathrm{~b})$. Here, we used the shifted laser and cold cavity frequencies $\omega_{1}^{\prime}=1 \gamma_{1}, \omega_{2}^{\prime}=1.01 \gamma_{1}, \Omega_{1}^{\prime}=1 \gamma_{1}$. In (b), we also show the time evolution of the real part of the field of the cold cavity $\operatorname{Re}\left[\mathrm{E}_{1}(\mathrm{t})\right]$. (c) Mean frequency of the laser oscillation $\bar{\omega}_{1,2}$ as a function of coupled strength $g_{1,2}$. (d) Mean frequency $\bar{\omega}_{1,2}$ for fixed coupling strength $\left(g_{1,2}=0.1 \gamma_{1}\right)$ but as a function of the resonance frequency of the cold cavity $\Omega_{1}$, which tunes the effective coupling strength between the two lasers.

lasers exhibit normal-mode splitting because they have no decay (gain). Note that, for Eqs. (6)-(8), anti-phase synchronization always occurs for any initial state, while if the signs of the two couplings are opposite such as $g_{2}=-g_{1}$, in-phase synchronization always occurs (not shown) The sign of a coupling constant depends on the overlap integral of cavity fields and may vary depending on the distance between cavities. In the device design in this paper, since all the distances between cavities are designed to be equal, all the signs of coupling constants can be assumed to be the same. In any case, the property that a synchronization phase does not depend on initial phases of lasers is of importance because the initial phase of PhC lasers cannot be controlled experimentally.

Synchronization tree. In Fig. 2c, we show the mean frequencies of the two laser oscillations $\bar{\omega}_{1}^{\prime}$ and $\bar{\omega}_{2}^{\prime}$ as a function of the coupling between cavities $g_{1,2}$. Since, in general, limit cycle oscillations are quasi-periodic when coupling strength is lower than the critical strength of synchronization, we need to use their mean frequencies obtained with peak detection. Figure $2 c$ clearly indicates that the mean frequencies symmetrically approach each other with an increase in the coupling strength $g$ and that they merge as $\bar{\omega}_{1}^{\prime}=\bar{\omega}_{2}^{\prime}=1.005 \gamma_{1}$ at the critical strength $g=0.05 \gamma_{1}$. In fact, the frequency $\bar{\omega}_{1,2}^{\prime}=1.005 \gamma_{1}$ is the mean frequency of $\omega_{1}^{\prime}=1 \gamma_{1}$ and $\omega_{2}^{\prime}=1.01 \gamma_{1}$ without coupling. Note that the synchronization tree shown in Fig. $2 \mathrm{c}$ is approximately symmetric for $\bar{\omega}_{1}^{\prime}$ and $\bar{\omega}_{2}^{\prime}$, which is because the parameters are almost the same for L1 and L2.

Furthermore, in Fig. 2d, we plot the mean frequency $\bar{\omega}_{1,2}^{\prime}$ as a function of the resonance frequency of the cold cavity $\Omega_{1}$, where the coupling strengths are fixed as $g_{1} \stackrel{2}{=} g_{2}=0.07 \gamma_{1}$ while $\Omega_{1}$ is swept from $\omega_{1}-1 \gamma_{1}$ to $\omega_{1}+1 \gamma_{1}$. Figure $2 \mathrm{~d}$ indicates that the effective coupling strengths between the cavities can be tuned by changing the resonance frequency of the cold cavity $\Omega_{1}$. Intuitively, as the cold-cavity's frequency deviates from the resonance frequencies of the two lasers, the effective coupling strengths decrease. In PhC cavities, the tuning of cavity coupling strength, which is determined by the distance between cavities, is almost impossible. Meanwhile the tuning of the cold cavity's resonance frequency is technically available with the carrier-injection ${ }^{61,62}$ or thermooptic techniques ${ }^{63,64}$, and thus the synchronization tree shown in Fig. $2 \mathrm{~d}$ could be measured.

\section{Phase equations of motion}

In this section, as we did in Ref. ${ }^{43}$, by performing the phase reduction analysis ${ }^{2,65}$ for Eqs. (6)-(8), we attempt to obtain phase equations of motion. In our case, the phase of limit cycle oscillation is nothing else but the phase of a laser $\phi$ as illustrated in Fig. 1a, and thus the interpretation of corresponding phase equations of motion is also straightforward. Furthermore, we show that the determination of phase equations of motion is of importance in terms of mapping our model to the local Kuramoto model. The price to pay for obtaining phase equations of motion is the adiabatic elimination of the field in cold-cavity $\mathrm{C} 1$, which is required to transform the indirectly coupled system to a directly coupled model with dissipative coupling.

Adiabatic elimination approximation. The adiabatic elimination of the cold-cavity field degree of freedom $\dot{E}_{1}=0$ requires that field $E_{1}$ rapidly decays compared with the laser field $\alpha_{1,2}$, and thus $E_{1}$ adiabatically follows $\alpha_{1}$ and $\alpha_{2}$. The time-scale of a variable is generally characterized by its decay rate. Therefore, the conventional adiabatic elimination of field $E_{1}$ requires that the decay rate $\Gamma_{1}$ must be larger than the decay rates of $\alpha_{1}$ and 
(a)

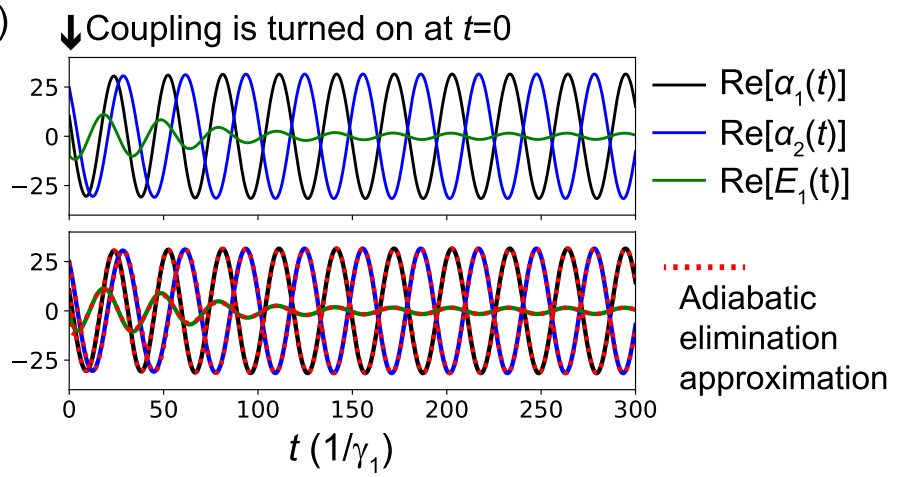

(b)

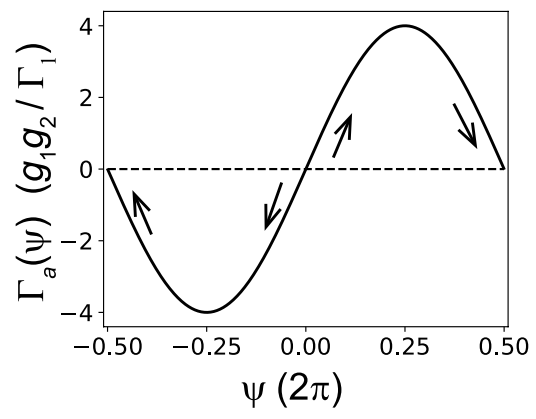

Figure 3. (a) Time evolution of the fields $\operatorname{Re}\left[\alpha_{1,2}(\mathrm{t})\right]$ and $\operatorname{Re}\left[\mathrm{E}(\mathrm{t})_{1}\right]$, but coupling $\left(g_{1,2}=0.1 \gamma_{1}\right)$ is turned on at $t=0$, which represents synchronization dynamics. The upper panel shows the time evolutions of the fields calculated with the original coupled-mode equations. In the lower panel, the time evolutions of the fields calculated with the adiabatic elimination approximation are shown as red dashed lines with the original plots. The shifted frequencies of the laser and cold cavities are $\omega_{1}^{\prime}=0.2 \gamma_{1}, \omega_{2}^{\prime}=0.21 \gamma_{1}$, and $\Omega_{1}^{\prime}=0.2 \gamma_{1}$. (b) Antisymmetric part of the phase coupling function $\Gamma_{a}(\psi)$ given by Eq. (16), where $\psi$ is the phase difference between the two laser phases defined as $\psi \equiv \phi_{2}-\phi_{1}$.

$\alpha_{2}$, as shown in "Lasers as limit cycle oscillators" section, which is not the case, for example, when we consider $\Gamma_{1}=\gamma_{1,2}$ as in Fig. 2. However, importantly, the time scale of the laser field $\alpha_{1,2}$ is not characterized solely by $\gamma_{1,2}$. Now, it is important to define the effective decay rates for $\alpha_{1}, \alpha_{2}$, and $E_{1}$, including both oscillation frequencies and pump parameters, as $\lambda_{1} \equiv-\gamma_{1} \epsilon_{1} / 2+i \omega_{1}, \lambda_{2} \equiv-\gamma_{2} \epsilon_{2} / 2+i \omega_{1}$, and $\Lambda_{1} \equiv \Gamma_{1} / 2+i \Omega_{1}$, respectively. Here, the oscillation frequencies $\omega_{1}, \omega_{2}$, and $\Omega_{1}$ are the imaginary parts of the effective decay rates. First, as pointed out by Haken ${ }^{56}$, to compare the time scales of the variables, in the effective decay rates, the imaginary parts must be negligible compared to the real parts: $\operatorname{Im}\left[\lambda_{1,2}\right] \ll \operatorname{Re}\left[\lambda_{1,2}\right]$ and $\operatorname{Im}\left[\Lambda_{1}\right] \ll \operatorname{Re}\left[\Lambda_{1}\right]$. Even though the cavity resonance frequencies $\omega_{1,2}$ and $\Omega_{1}$ are always much higher than the terms $\gamma_{1,2} \epsilon_{1,2} / 2$ and $\Gamma_{1} / 2$, if all the resonance frequencies of the cavities have similar values $\omega_{1} \simeq \omega_{2} \simeq \Omega_{1}$, the imaginary parts in the effective decay rates become negligible in a rotating frame with the frequency of $\Omega_{1}$. Second, by comparing the real parts of the effective decay rates $\operatorname{Re}\left[\lambda_{1,2}\right]$ and $\operatorname{Re}\left[\Lambda_{1}\right]$, we find that the sign of $\operatorname{Re}\left[\Lambda_{1}\right]$ is always positive, while the sign of $\operatorname{Re}\left[\lambda_{1,2}\right]$ can be negative due to gain when the pump power is above the threshold $\varepsilon_{1,2} \geq 0$. According to Ref. ${ }^{56,66}$, when $\operatorname{Re}\left[\Lambda_{1}\right]>0$ and $\operatorname{Re}\left[\lambda_{1,2}\right] \leq 0$, the field $E_{1}$ is a "stable" mode that rapidly decays, while the laser fields $\alpha_{1}$ and $\alpha_{2}$ are unstable modes that do not decay but govern the slow dynamics of the system, which allows putting $\dot{E}_{1}=0$ (adiabatic elimination). In fact, the unstable mode $\alpha_{1,2}$ "enslaves" the stable mode $E_{1}$ and plays a role as an "order parameter" (the slaving principle ${ }^{56,66}$ ).

Now, setting $\dot{E}_{1}=0$ for Eq. (7), we eliminate the cold-cavity field degree of freedom as

$$
E_{1}=-i \frac{2}{\Gamma_{1}}\left(g_{1} \alpha_{1}+g_{2} \alpha_{2}\right)
$$

By substituting Eq. (9) into Eqs. (6) and (8), we obtain approximated equations of motion:

$$
\begin{aligned}
& \dot{\alpha}_{1}=-i \omega_{1} \alpha_{1}+\left[\frac{1}{2} \gamma_{1} \varepsilon_{1}-\frac{2 g_{1}^{2}}{\Gamma_{1}}\right] \alpha_{1}-\frac{1}{2} \beta_{1} \gamma_{1}\left|\alpha_{1}\right|^{2} \alpha_{1}-\frac{2 g_{1} g_{2}}{\Gamma_{1}} \alpha_{2} \\
& \dot{\alpha}_{2}=-i \omega_{2} \alpha_{2}+\left[\frac{1}{2} \gamma_{2} \varepsilon_{2}-\frac{2 g_{2}^{2}}{\Gamma_{1}}\right] \alpha_{2}-\frac{1}{2} \beta_{2} \gamma_{2}\left|\alpha_{2}\right|^{2} \alpha_{2}-\frac{2 g_{1} g_{2}}{\Gamma_{1}} \alpha_{1} .
\end{aligned}
$$

To confirm the validity of this adiabatic elimination approximation, in Fig. 3a, we show synchronization dynamics calculated both with the original equations of motion (6)-(8) and approximated equations (10) and (11). In Fig. 3a, coupling with $g_{1,2}=0.1 \gamma_{1}$ is switched on at $t=0$ for uncoupled steady-state laser oscillations, and thus the time evolutions of fields represent synchronization dynamics from the unsynchronized to synchronized state. The upper panel in Fig. 3a shows only the synchronization dynamics calculated with the original equations of motion (6)-(8). Meanwhile, in the lower panel, synchronizations calculated with the original equations of motion (solid lines) overlap those calculated with the approximated equations of motion (dashed lines), which clearly indicates that two time evolutions are almost indistinguishable and that the adiabatic elimination approximation is surprisingly good. Note that, to clearly show the synchronization dynamics in Fig. 3a, we used shifted frequencies $\omega_{1}^{\prime}=0.2 \gamma_{1}, \omega_{2}^{\prime}=\omega_{1}^{\prime}+\Delta \omega=0.21 \gamma_{1}$ and $\Omega_{1}^{\prime}=\omega_{1}^{\prime}=0.2 \gamma_{1}$, which are lower than those Fig. 1 . As we commented in "Coupled-mode equations" section, these shifts of the resonance frequencies do not change the physics, because only the relative relationship between the resonance frequencies is important. Since the field in the cold cavity was adiabatically eliminated, Eqs. (10) and (11) represent directly coupled lasers. Furthermore, in Eqs. (10) and (11), the effective couplings represented by $-\left(2 g_{1} g_{2} / \Gamma_{1}\right) \alpha_{2}$ and $-\left(2 g_{1} g_{2} / \Gamma_{1}\right) \alpha_{1}$ are non-energyconserving dissipative couplings, which intuitively explains why normal-mode splitting does not appear in our model. Additionally, in Eqs. (10) and (11), the effective dissipative coupling does not have the time delay. 
Finally, we comment on synchronization with a large coupling strength. We found that Eqs. (10) and (11) fail to reproduce synchronization dynamics when $g_{1,2} \geq \gamma_{1,2}, \Gamma_{1}$, which is because the adiabatic elimination approximation cannot describe coherent intensity oscillation between cavities associated with this parameter region [see Section 2 in the Supplemental Material (SM)]. Therefore, the complete conditions required for the adiabatic elimination approximation are

$$
\omega_{1} \simeq \omega_{2} \simeq \Omega_{1} \text { and } g_{1,2}<\gamma_{1,2}, \Gamma_{1} .
$$

Here, it is also important to stress that, although the adiabatic elimination fails to describe synchronization dynamics, even when $g_{1,2} \geq \gamma_{1,2}, \Gamma_{1}$, stable synchronization itself can occur and the adiabatic elimination approximation well reproduces the steady-state synchronized oscillations (see Section 2 in the SM). Furthermore, even when the coupling is extremely strong, for example, $g_{1,2}=10 \gamma_{1}$, we can observe stable synchronization, where no normal-mode splitting is present (not shown). This insensitivity to coupling strength will be advantageous in terms of real device designs, because adjusting the value of weak coupling strength is technically difficult ${ }^{43}$. Furthermore, if coupling is sufficiently strong, we may prove synchronization from spectral shapes, which is discussed again in "Discussion" section.

Phase reduction analysis. Now, we perform the phase reduction analysis for equations of motion (10) and (11), which were obtained with the adiabatic elimination approximation. Here, we make use of the consequence of the phase reduction theory without going into the theoretical detail, which is briefly provided in Section 1 in the SM (further details can be found in our recent paper ${ }^{43}$ and in $\operatorname{Refs}^{2,44}$ ). The objective of the phase reduction analysis is to obtain the phase equations of motion for the phases of the laser L1 $\left(\phi_{1}\right)$ and L2 $\left(\phi_{2}\right)$ represented as

$$
\begin{aligned}
& \dot{\phi_{1}}=-\omega_{1}+\Gamma_{12}\left(\phi_{1}-\phi_{2}\right) \\
& \dot{\phi_{2}}=-\omega_{2}+\Gamma_{21}\left(\phi_{2}-\phi_{1}\right),
\end{aligned}
$$

where $\Gamma_{12}(\phi)$ and $\Gamma_{21}(\phi)$ are called the phase-coupling functions. For the approximated equations of motion (10) and (11), we found that $\Gamma_{12}(\phi)$ and $\Gamma_{21}(\phi)$ can be analytically calculated as

$$
\Gamma_{12}(\theta)=\Gamma_{21}(\theta)=\frac{2 g_{1} g_{2}}{\Gamma_{1}} \sin \theta
$$

Finally, the phase difference between the two lasers $\psi \equiv \phi_{2}-\phi_{1}$ follows the following simple equation of motion:

$$
\dot{\psi}=-\Delta \omega+\Gamma_{a}(\psi) \text { with } \Gamma_{\mathrm{a}}(\psi)=\frac{4 \mathrm{~g}_{1} \mathrm{~g}_{2}}{\Gamma_{1}} \sin \psi,
$$

where $\Delta \omega \equiv \omega_{2}-\omega_{1}$ is the frequency difference between the two lasers already defined in "Coupled-mode equations" section. Here, $\Gamma_{a}(\psi) \equiv \Gamma_{21}(\psi)-\Gamma_{12}(-\psi)$ is the anti-symmetric part of the phase coupling function $\Gamma_{21}(\psi)$, which is shown in Fig. 3b. For a negligible laser frequency difference $\Delta \omega \simeq 0$, since $\Gamma_{a}(\pi)=0$ and $\Gamma_{a}^{\prime}(\pi)<0$ hold for Eq. (16), phase locking occurs at the phase $\psi=\phi_{2}-\phi_{1}=\pi$, which is anti-phase synchronization as expected from the simulations [see the arrows in Fig. 3b]. Meanwhile, since $\Gamma_{a}(0)=0$ and $\Gamma_{a}^{\prime}(0)>0$ hold for $\phi=0$, the phase $\phi=0$ is an unstable fixed point. Of course, for a non-negligible frequency difference $\Delta \omega \neq 0$, the synchronization phase shifts from $\pi$. The phase equations of motion predict not only the synchronization phase but also the critical coupling strength of synchronization. For Eq. (16) to have a phase-locking solution, the condition $-4 g_{1} g_{2} / \Gamma_{1} \leq \Delta \omega \leq 4 g_{1} g_{2} / \Gamma_{1}$ must be satisfied. For the oscillation frequency difference $\Delta \omega=0.01 \gamma_{1}$ and cold-cavity decay rate $\Gamma_{1}=1 \gamma_{1}$, which are assumed in Fig. 2 c, synchronization occurs when the coupling strengths reach $g_{1}=g_{2}=0.05 \gamma_{1}$ [see Fig. 2c] because the above phase-locking condition is satisfied with these parameters as $4 g_{1} g_{2} / \Gamma_{1}=0.01 \gamma_{1}=\Delta \omega$.

Furthermore, the analytically calculated phase coupling functions in Eq. (15) are also of importance for mapping our model to the local Kuramoto model. In fact, the phase equations of motion are explicitly written as

$$
\begin{gathered}
\dot{\phi}_{1}=-\omega_{1}+\tilde{g}_{12} \sin \left(\phi_{1}-\phi_{2}\right) \\
\dot{\phi}_{2}=-\omega_{2}+\tilde{g}_{21} \sin \left(\phi_{2}-\phi_{1}\right),
\end{gathered}
$$

where $\tilde{g}_{i j} \equiv 2 g_{i} g_{j} / \Gamma_{1}\left(\tilde{g}_{i j}=\tilde{g}_{j i}\right)$ is the effective coupling strength. The phase equations of motion (17) and (18) are straightforwardly extended to a one-dimensional chain or two-dimensional array as

$$
\dot{\phi}_{i}=-\omega_{i}+\sum_{j \in N_{i}} \tilde{g}_{i j} \sin \left(\phi_{i}-\phi_{j}\right)
$$

where $N_{j}$ represents the nearest neighbour sites of the $i$ th site. Importantly, the coupled phase oscillator described by Eq. (19) is equivalent to the local Kuramoto model ${ }^{3,25}$. Note that, in the original Kuramoto model, the sign of the coupling is minus as $-\tilde{g}_{21}$, and thus in-phase synchronization occurs.

In conclusion, with the aide of the phase reduction theory, we proved that an array of lasers with cold-cavitymediated coupling can emulate the nearest-neighbor coupled Kuramoto model (the local Kuramoto model). Note that, of course, the strict mapping of given coupled-mode equations to the local Kuramoto model (19) requires an adiabatic elimination condition similar to Eq. (12). 
(a)
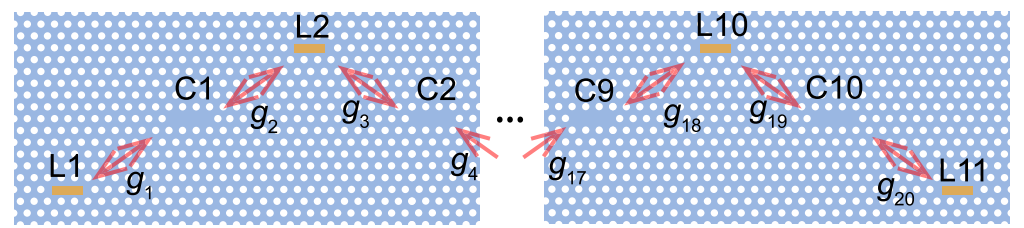

(b)

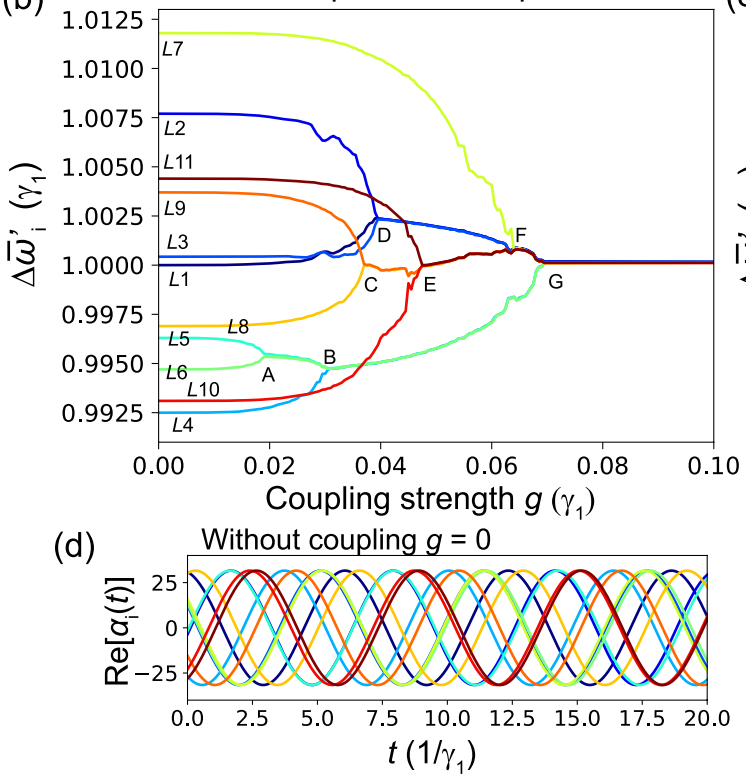

(c)
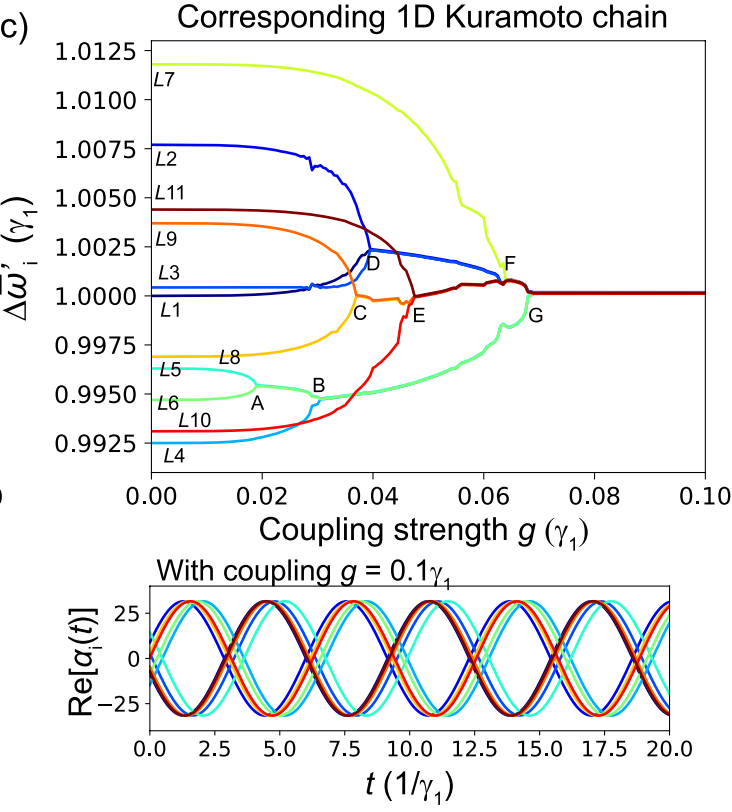

Figure 4. (a) Schematic of a chain of eleven indirectly coupled $\mathrm{PhC}$ lasers that emulates the Kuramoto chain. Indices $\mathrm{L} i$ and $\mathrm{C} i$ represent the $i$ th laser and cold cavities, respectively. The shifted resonance frequencies of the laser cavities are $\omega_{1}^{\prime}=1.0000, \omega_{2}^{\prime}=1.0077, \omega_{3}^{\prime}=1.0004, \omega_{4}^{\prime}=0.9925, \omega_{5}^{\prime}=0.9963, \omega_{6}^{\prime}=0.9947, \omega_{7}^{\prime}=1.0118$, $\omega_{8}^{\prime}=0.9969, \omega_{9}^{\prime}=1.0037, \omega_{10}^{\prime}=0.9931$, and $\omega_{11}^{\prime}=1.0044$, where the units are $\gamma_{1}$. For the other parameters, we use $\beta_{i}=0.001, \varepsilon_{i}=1.0, \gamma_{i} \equiv 1, \Omega_{i}=1 \gamma_{1}$ and $\Gamma_{i}=\gamma_{1} \equiv 1$ for all $i$. (c) Synchronization tree calculated with the local Kuramoto chain (Eq. (19)) corresponding to (b). (b) The mean oscillation frequencies of the eleven lasers $\bar{\omega}_{i}^{\prime}$ are shown as a function the coupling strength $g_{i}=g$ for all $i$. The synchronization points are denoted by A-G. (d) Time evolutions of the real parts of the fields in all the laser cavities without $g=0$ (left) and with coupling $g=0.1 \gamma_{1}$ (right).

\section{Array configuration}

Although the investigation of rich physics emerging from coupled phase oscillators is beyond the scope of this paper, we briefly simulate a one-dimensional chain of indirectly coupled $\mathrm{PhC}$ lasers and demonstrate that our device can actually reproduce collective dynamics predicted for the one-dimensional local Kuramoto chain ${ }^{46}$. The chain of indirectly coupled PhC lasers is schematically illustrated in Fig. 4a, where eleven laser cavities and ten cold cavities are alternately aligned. Of course, the configuration of cavities to realize the local Kuramoto chain is not limited to that shown in Fig. 4 a, and various configurations can be imagined. For a one-dimensional chain, in principle, even the periodic boundary condition may be implemented with a ring-like configuration. For simplicity, for all the laser cavities, we assume $\beta_{i}=0.001, \varepsilon_{i}=1.0$, and $\gamma_{i} \equiv 1$. Similarly, all the cold cavities have the same resonance frequencies and photon decay rate: $\Omega_{i}=1 \gamma_{1}$ and $\Gamma_{i}=\gamma_{1} \equiv 1$ for all $i$. In Section 6 in the supplemental material, we demonstrate large-scale synchronization when the parameter values of all laser and cold cavities are slightly different. Furthermore, as in "Synchronization of two lasers" section, we assume that all the coupling constants have the same strengths: $g_{i}=g$ for all $i$. Meanwhile, the resonance frequencies of the eleven laser cavities are randomly distributed around a mean frequency $\bar{\omega}_{i}=1 \gamma_{1}$ [for the actual values of $\omega_{i}$, please see the caption of Fig. 4]. Note that since all the laser and cold cavities have similar resonance frequencies and the coupling strengths are smaller than the cavity decay rates, an adiabatic elimination condition similar to Eq. (12) is satisfied, and thus corresponding simple phase equations of motion are expected to exist.

By directly simulating the full coupled-mode equations corresponding to the configuration shown in Fig. 4a, we calculated the mean frequencies of the laser oscillations as a function of the coupling strength $g$ [see the synchronization tree in Fig. 4b]. As Fig. 4b indicates, with an increase in coupling strength $g$, synchronized clusters are gradually formed, and finally all clusters merge into a single fully synchronized cluster at $g \simeq 0.07 \gamma_{1}$ [see $\mathrm{G}$ on Fig. 4b]. Similarly to Ref. ${ }^{46}$, when two [at A, B, C in Fig. 4b] or three [at D, E in Fig. 4b] adjacent oscillators (or clusters) have close oscillation frequencies, they form a new synchronized cluster with an increase in coupling strength. When adjacent clusters have largely different frequencies, while non-adjacent clusters have similar frequencies, the non-adjacent clusters form a synchronized cluster. In fact, the synchronization denoted by $\mathrm{F}$ in Fig. $4 \mathrm{~b}$ consists of the non-adjacent oscillators (clusters) L1-3 and L7-11. Furthermore, in Fig. 4c, we show a 
synchronization tree calculated with the local Kuramoto chain (Eq. 19) corresponding to Fig. 4 b. The fact that both synchronization trees have almost the same structures indicates that our proposed device will actually emulate the local Kuramoto model.

Finally, the time evolutions of the laser oscillations without $(g=0)$ and with coupling $\left(g=0.1 \gamma_{1}\right)$ are shown in the left and right panels of Fig. $4 \mathrm{~d}$, respectively. When there is no coupling, as we expect, the laser oscillations are totally uncorrelated, while all the laser oscillations are fully synchronized with coupling $g=0.1 \gamma_{1}$. Interestingly, in this fully synchronized state [see the right panel in Fig. 4d], the phases are opposite between the even and odd sites of the lasers oscillations. Therefore, even in the one-dimensional chain, a pair of adjacent laser oscillations exhibit anti-phase synchronization. Note that, in Fig. 4c,d, the "de-synchronization" discovered in ${ }^{46}$ was not observed, which may be due to the small number of oscillators or, more interestingly, could be associated with anti-phase synchronization. We also comment on the offsets of the synchronization phases in the fully-synchronized oscillations shown in the right panel of Fig. $4 \mathrm{~d}$, where the synchronization phases slightly differ depending on the pair of the synchronized oscillations. We found that, with a further increase in coupling strength, these offsets of the synchronization phases disappear and that all the pairs of synchronized oscillations become indistinguishable.

\section{Discussion}

Here, we discuss several details that will be of importance in a real device design and experiments. In experiments, the easiest method to observe synchronization may be the spectral measurement of laser emissions. Since limit cycle oscillation frequencies are equivalent to laser oscillation frequencies, synchronization can be directly confirmed by the number of emission peaks in a measured spectrum. Namely, if a spectrum has a single emission peak, two lasers are synchronized, while if there are two emission peaks, they are not. Although coupling strengths between cavities are usually fixed in a device, it is still possible to actively tune the resonance frequency of a cold cavity ${ }^{61-64,67}$ and effectively change coupling strengths as shown in Fig. $2 \mathrm{~d}$. In this context, the spectral shape of laser emissions will be of interest. Below the lasing threshold, since laser cavities will behave as "cold cavities", their emission spectrum is expected to exhibit normal-mode splitting. Meanwhile, above the lasing threshold the emission spectrum exhibits a single peak due to synchronization. Therefore, we may prove synchronization from the pump-power dependence of the change in spectral shape. Another promising experimental strategy to prove synchronization may be to pump two lasers independently and tune the respective laser frequencies by making use of the carrier-induced blue shift ${ }^{49}$. This strategy can be easily realized with spatially separated optical pumps or two electrodes for electric pumping.

The buried MQW PhC laser technique in the design of a real device is reported in Refs. ${ }^{47,48}$, where the $\mathrm{PhC}$ slab and buried $\mathrm{PhC}$ are composed of InP and InGaAsP/InGaAs, respectively. Furthermore, buried MQW PhC lasers can be pumped optically or electrically. If the photon lifetime of buried MQW PhC lasers is assumed to be $1 / \gamma_{1}=1$ ps $(\sim 160 \mathrm{GHz})$, the frequency difference between two lasers corresponding to $\Delta \omega=0.01 \gamma_{1}$, which is assumed in the simulations in Fig. 2 , is $\Delta \omega=0.01 \gamma_{1} \sim 1.6 \mathrm{GHz}$. This laser frequency difference may seem to be severe for experimental realization (even with the state-of-the-art fabrication technology, the frequency difference between cavities may be about $50 \mathrm{GHz}^{68}$ ), but we found that, qualitatively, the same synchronization can occur for a larger frequency difference. For example, synchronization with a laser frequency difference $\Delta \omega=0.1 \gamma_{1}$ is discussed in Section 3 in the SM. Furthermore, we found that even if all the parameters of the three cavities including $\beta_{1,2}$ are moderately different, synchronization can occur (not shown).

\section{Conclusion and outlook}

To conclude, we theoretically proposed a design of indirectly-coupled $\mathrm{PhC}$ cavity lasers that emulates the local Kuramoto model. In this study, we reinterpreted the injection-locking phenomenon of lasers as the synchronization of limit cycle oscillations. Furthermore, our design prevents laser oscillations from forming normal-modes (strong-coupling) with indirect coupling via additional cold cavities and realizes effective dissipative coupling without time-delay. Experimentally, this proposed structure will best be realized best by using buried MQW PhC cavities. First, after modelling laser oscillation with the Stuart-landau equation, we numerically demonstrated the synchronization of two indirectly-coupled PhC lasers using the coupled-mode equations of motion. Second, by applying the phase reduction theory to the two indirectly coupled lasers, we obtained corresponding phase equations of motion, which are equivalent to the local Kuramoto model. Finally, we briefly discussed synchronization dynamics for a one-dimensional chain of indirectly coupled $\mathrm{PhC}$ lasers and demonstrated that the proposed device can actually emulate the local Kuramoto chain.

For future perspectives, first of all, the one-dimensional local Kuramoto model briefly investigated in "Array configuration" section, already comprises rich physics that were actively investigated by detailed numerical simulations ${ }^{46}$ and renormalization group analysis ${ }^{25,69}$. Furthermore, very recently, Ref. ${ }^{70}$ demonstrated that even topological phenomena emerge in the one-dimensional chain of limit cycles. Thanks to the scalability of PhC cavities, the extension of the one-dimensional chain of $\mathrm{PhC}$ lasers to a two-dimensional array is straightforward, which is the realization of the celebrated two-dimensional local Kuramoto model ${ }^{3,24-28,71}$. Compared with the in-phase synchronization case, large-scale anti-phase synchronization has not yet been drawing attention. For instance, as Ref. ${ }^{72}$ indicates that a large anti-phase synchronization network is not possible, large-scale anti-phase synchronization itself may be of fundamental interest. Another important direction will be the inclusions of classical and quantum noise effects in indirectly coupled PhC lasers, which will provide spectral information. As we briefly discussed in "Discussion" section, we may prove synchronization in terms of the pump power dependence of spectral shape. In this direction, it is also easy to construct a quantum model corresponding to our coupled-mode equations. In fact, the quantum counterpart of the classical Stuart-Landau model is the Scully-lamb master equation ${ }^{73,74}$. Therefore, even the effect of quantum noises on synchronization ${ }^{21,58,75}$ may 
be tested with the proposed device. Moreover, since the synchronization problem in the local Kuramoto model is analogous to the energy minimization problem in the XY model, our device may be used for simulating the spin system in statistical physics ${ }^{72,76}$. Finally, from the standpoint of practical application, an injection-locked (synchronized) $\mathrm{PhC}$ laser array can be employed as a single-mode high-power PhC laser. Even though every $\mathrm{PhC}$ laser unavoidably has a different oscillation frequency, in the fully synchronized state, they behave as a laser with a single frequency. Furthermore, this type of a laser will also have high coherence because all laser phases are locked in the synchronized state.

\section{Methods}

All the time evolutions were obtained by integrating the coupled-mode equations of motion with the conventional Runge-Kutta method. The synchronization trees were calculated as the mean oscillation frequencies of time evolutions. The calculation of the mean frequencies is based on the peak detection technique. To precisely determine the mean frequencies, long time evolutions (typically $6000 \gamma_{1}^{-1}$ ) were required.

Received: 30 October 2020; Accepted: 1 March 2021

Published online: 21 April 2021

\section{References}

1. Pikovsky, A., Kurths, J., Rosenblum, M. \& Kurths, J. Synchronization: A Universal Concept in Nonlinear Sciences Vol. 12 (Cambridge University Press, 2003).

2. Kuramoto, Y. Chemical Oscillations, Waves, and Turbulence (Courier Corporation, 2003).

3. Acebrón, J. A., Bonilla, L. L., Pérez Vicente, C. J., Ritort, F. \& Spigler, R. The kuramoto model: a simple paradigm for synchronization phenomena. Rev. Mod. Phys. 77, 137-185. https://doi.org/10.1103/RevModPhys.77.137 (2005).

4. Tsang, K. Y., Mirollo, R. E., Strogatz, S. H. \& Wiesenfeld, K. Dynamics of a globally coupled oscillator array. Phys. D Nonlinear Phenom. 48, 102-112. https://doi.org/10.1016/0167-2789(91)90054-D (1991).

5. Wiesenfeld, K., Colet, P. \& Strogatz, S. H. Synchronization transitions in a disordered josephson series array. Phys. Rev. Lett. 76, 404-407. https://doi.org/10.1103/PhysRevLett.76.404 (1996).

6. Barbara, P., Cawthorne, A. B., Shitov, S. V. \& Lobb, C. J. Stimulated emission and amplification in josephson junction arrays. Phys. Rev. Lett. 82, 1963-1966. https://doi.org/10.1103/PhysRevLett.82.1963 (1999).

7. Winful, H. G. \& Wang, S. S. Stability of phase locking in coupled semiconductor laser arrays. Appl. Phys. Lett. 53, $1894-1896$. https://doi.org/10.1063/1.100363 (1988).

8. Wang, S. S. \& Winful, H. G. Dynamics of phase locked semiconductor laser arrays. Appl. Phys. Lett. 52, 1774-1776. https://doi. org/10.1063/1.99622 (1988).

9. Thornburg, K. S. et al. Chaos and coherence in coupled lasers. Phys. Rev. E. 55, 3865-3869. https://doi.org/10.1103/PhysRevE.55. 3865 (1997).

10. Hohl, A., Gavrielides, A., Erneux, T. \& Kovanis, V. Quasiperiodic synchronization for two delay-coupled semiconductor lasers. Phys. Rev. A. 59, 3941-3949. https://doi.org/10.1103/PhysRevA.59.3941 (1999).

11. Kozyreff, G., Vladimirov, A. G. \& Mandel, P. Global coupling with time delay in an array of semiconductor lasers. Phys. Rev. Lett. 85, 3809-3812. https://doi.org/10.1103/PhysRevLett.85.3809 (2000).

12. Allaria, E., Arecchi, F. T., Di Garbo, A. \& Meucci, R. Synchronization of homoclinic chaos. Phys. Rev. Lett. 86, 791-794. https:// doi.org/10.1103/RevModPhys.77.1370 (2001).

13. Rogister, F., Thornburg, K. S., Fabiny, L., Möller, M. \& Roy, R. Power-law spatial correlations in arrays of locally coupled lasers. Phys. Rev. Lett. 92, $093905 . \quad$ https://doi.org/10.1103/PhysRevLett.92.093905 (2004).

14. Utsunomiya, S., Takata, K. \& Yamamoto, Y. Mapping of ising models onto injection-locked laser systems. Opt. Express 19, 1809118108. https://doi.org/10.1364/OE.19.018091 (2011).

15. Takata, K., Utsunomiya, S. \& Yamamoto, Y. Transient time of an ising machine based on injection-locked laser network. New J. Phys. 14, 013052. https://doi.org/10.1088/1367-2630/14/1/013052 (2012).

16. Utsunomiya, S. et al. Binary phase oscillation of two mutually coupled semiconductor lasers. Opt. Express 23, 6029-6040. https:// doi.org/10.1364/OE.23.006029 (2015).

17. Sun, Y., Combrié, S., Bretenaker, F. \& De Rossi, A. Mode locking of the hermite-gaussian modes of a nanolaser. Phys. Rev. Lett. 123, 233901. https://doi.org/10.1103/PhysRevLett.123.233901 (2019).

18. Baas, A. et al. Synchronized and desynchronized phases of exciton-polariton condensates in the presence of disorder. Phys. Rev. Lett. 100, 170401. https://doi.org/10.1103/PhysRevLett.100.170401 (2008).

19. Zhang, M. et al. Synchronization of micromechanical oscillators using light. Phys. Rev. Lett. 109, 233906. https://doi.org/10.1103/ PhysRevLett.109.233906 (2012).

20. Bagheri, M., Poot, M., Fan, L., Marquardt, F. \& Tang, H. X. Photonic cavity synchronization of nanomechanical oscillators. Phys. Rev. Lett. 111, 213902. https://doi.org/10.1103/PhysRevLett.111.213902 (2013).

21. Walter, S., Nunnenkamp, A. \& Bruder, C. Quantum synchronization of a driven self-sustained oscillator. Phys. Rev. Lett. 112, 094102. https://doi.org/10.1103/PhysRevLett.112.094102 (2014).

22. Ohadi, H. et al. Nontrivial phase coupling in polariton multiplets. Phys. Rev. X. 6, 031032. https://doi.org/10.1103/PhysRevX.6. 031032 (2016).

23. Xu, D. et al. Synchronization and temporal nonreciprocity of optical microresonators via spontaneous symmetry breaking. Adv. Photon. 1, 1-6. https://doi.org/10.1117/1.AP.1.4.046002 (2019).

24. Sakaguchi, H., Shinomoto, S. \& Kuramoto, Y. Local and grobal self-entrainments in oscillator lattices. Prog. Theor. Phys. 77, 1005-1010. https://doi.org/10.1143/PTP.77.1005 (1987).

25. Daido, H. Lower critical dimension for populations of oscillators with randomly distributed frequencies: a renormalization-group analysis. Phys. Rev. Lett. 61, 231-234. https://doi.org/10.1103/PhysRevLett.61.231 (1988).

26. Strogatz, S. H. \& Mirollo, R. E. Collective synchronisation in lattices of nonlinear oscillators with randomness. J. Phys. A Math. Gen. 21, L699-L705. https://doi.org/10.1088/0305-4470/21/13/005 (1988).

27. Hong, H., Park, H. \& Choi, M. Y. Collective synchronization in spatially extended systems of coupled oscillators with random frequencies. Phys. Rev. E. 72, 036217. https://doi.org/10.1103/PhysRevE.72.036217 (2005).

28. Lee, T. E., Tam, H., Refael, G., Rogers, J. L. \& Cross, M. C. Vortices and the entrainment transition in the two-dimensional kuramoto model. Phys. Rev. E. 82, 036202. https://doi.org/10.1103/PhysRevE.82.036202 (2010).

29. Altug, H. \& Vučković, J. Two-dimensional coupled photonic crystal resonator arrays. Appl. Phys. Lett. 84, 161-163. https://doi. org/10.1063/1.1639505 (2004). 
30. Altug, H. \& Vučković, J. Photonic crystal nanocavity array laser. Opt. Express 13, 8819-8828. https://doi.org/10.1364/OPEX.13. 008819 (2005).

31. Hamel, P. et al. Spontaneous mirror-symmetry breaking in coupled photonic-crystal nanolasers. Nat. Photon. 9, 311-315. https:// doi.org/10.1038/nphoton.2015.65 (2015).

32. Takata, K. \& Notomi, M. $\mathcal{P} \mathcal{T}$-symmetric coupled-resonator waveguide based on buried heterostructure nanocavities. Phys. Rev. Appl. 7, 054023. https://doi.org/10.1103/PhysRevApplied.7.054023 (2017).

33. Marconi, M. et al. Far-from-equilibrium route to superthermal light in bimodal nanolasers. Phys. Rev. X. 8, 011013. https://doi. org/10.1103/PhysRevX.8.011013 (2018).

34. Takata, K. et al. Observing exceptional point degeneracy of radiation with electrically pumped photonic crystal coupled-nanocavity lasers. Optica. 8, 184-192. https://doi.org/10.1364/OPTICA.412596 (2021).

35. Noda, S., Kojima, K. \& Kyuma, K. Mutual injection-locking properties of monolithically-integrated surface-emitting multiplequantum-well distributed feedback lasers. IEEE J. Quant. Electron. 26, 1883-1894. https://doi.org/10.1109/3.62107 (1990).

36. Chan, L. Y. et al. Demonstration of an all-optical switch by use of a multiwavelength mutual injection-locked laser diode. Opt. Lett. 28, 837-839. https://doi.org/10.1364/OL.28.000837 (2003).

37. Tauke-Pedretti, A. et al. Mutual injection locking of monolithically integrated coupled-cavity dbr lasers. IEEE Photon. Technol. Lett. 23, 908-910. https://doi.org/10.1109/LPT.2011.2140099 (2011).

38. Kurtz, R. M. et al. Mutual injection locking: a new architecture for high-power solid-state laser arrays. IEEE J. Sel. Top. Quant. Electron. 11, 578-586 (2005).

39. Chen, Z., Hou, J., Zhou, P. \& Jiang, Z. Mutual injection-locking and coherent combining of two individual fiber lasers. IEEE J. Quant. Electron. 44, 515-519. https://doi.org/10.1109/JQE.2008.917966 (2008).

40. Sun, C. et al. Modulation characteristics enhancement of monolithically integrated laser diodes under mutual injection locking. IEEE J. Sel. Top. Quant. Electron. 21, 628-635. https://doi.org/10.1109/JSTQE.2015.2478817 (2015).

41. Kobayashi, S. \& Kimura, T. Injection locking in algaas semiconductor laser. IEEE J. Quant. Electron. 17, 681-689 (1981).

42. Siegman, A. E. Lasers (University Science Mill Valley, 1986).

43. Takemura, N., Takiguchi, M. \& Notomi, M. Designs toward synchronization of optical limit cycles with coupled silicon photonic crystal microcavities. Opt. Express 28, 27657-27675. https://doi.org/10.1364/OE.399545 (2020).

44. Nakao, H. Phase reduction approach to synchronisation of nonlinear oscillators. Contemp. Phys. 57, 188-214. https://doi.org/10. 1080/00107514.2015.1094987 (2016).

45. Stankovski, T., Pereira, T., McClintock, P. V. \& Stefanovska, A. Coupling functions: Universal insights into dynamical interaction mechanisms. Rev. Mod. Phys. 89, 045001. https://doi.org/10.1103/RevModPhys.89.045001 (2017).

46. Zheng, Z., Hu, G. \& Hu, B. Phase slips and phase synchronization of coupled oscillators. Phys. Rev. Lett. 81, 5318-5321. https:// doi.org/10.1103/PhysRevLett.81.5318 (1998).

47. Takeda, K. et al. Few-fj/bit data transmissions using directly modulated lambda-scale embedded active region photonic-crystal lasers. Nat. Photon. 7, 569-575. https://doi.org/10.1038/nphoton.2013.110 (2013).

48. Matsuo, S. et al. Ultralow operating energy electrically driven photonic crystal lasers. IEEE J. Sel. Top. Quant. Electron. 19, 49003114900311. https://doi.org/10.1109/JSTQE.2013.2249048 (2013).

49. Takiguchi, M. et al. Systematic study of thresholdless oscillation in high- $\beta$ buried multiple-quantum-well photonic crystal nanocavity lasers. Opt. Express 24, 3441-3450. https://doi.org/10.1364/OE.24.003441 (2016).

50. Takemura, N. et al. Lasing thresholds and photon statistics in high- $\beta$ buried multiple quantum well photonic crystal nanocavity lasers. Phys. Rev. A. 99, 053820. https://doi.org/10.1103/PhysRevA.99.053820 (2019).

51. Rice, P. R. \& Carmichael, H. J. Photon statistics of a cavity-qed laser: a comment on the laser-phase-transition analogy. Phys. Rev. A. 50, 4318-4329. https://doi.org/10.1103/PhysRevA.50.4318 (1994).

52. van Druten, N. J. et al. Laser with thresholdless intensity fluctuations. Phys. Rev. A. 62, 053808. https://doi.org/10.1103/PhysRevA. 62.053808 (2000).

53. Lariontsev, E. G. Nonlinear features of quantum fluctuations in a slow-inversion laser. Phys. Rev. A. 83, 063803. https://doi.org/ 10.1103/PhysRevA.83.063803 (2011).

54. Arecchi, F. T. \& Harrison, R. G. Instabilities and Chaos in Quantum Optics Vol. 34 (Springer Science \& Business Media, 2012).

55. Louisell,. Quantum Statistical Properties of Radiation Vol. 7 (Wiley, 1973).

56. Haken, H. Synergetics (Springer, 1977).

57. Strogatz, S. H. Nonlinear Dynamics and Chaos: With Applications to Physics, Biology, Chemistry, and Engineering (CRC Press, 2018).

58. Lee, T. E. \& Sadeghpour, H. R. Quantum synchronization of quantum van der pol oscillators with trapped ions. Phys. Rev. Lett. 111, 234101. https://doi.org/10.1103/PhysRevLett.111.234101 (2013).

59. Takemura, N., Omachi, J. \& Kuwata-Gonokami, M. Fast periodic modulations in the photon correlation of single-mode verticalcavity surface-emitting lasers. Phys. Rev. A. 85, 053811. https://doi.org/10.1103/PhysRevA.85.053811 (2012).

60. Wang, T., Puccioni, G. \& Lippi, G. Dynamical buildup of lasing in mesoscale devices. Sci. Rep. 5, 15858 (2015).

61. Fushman, I. et al. Ultrafast nonlinear optical tuning of photonic crystal cavities. Appl. Phys. Lett. 90, 091118. https://doi.org/10. $1063 / 1.2710080$ (2007).

62. Tanabe, T., Nishiguchi, K., Kuramochi, E. \& Notomi, M. Low power and fast electro-optic silicon modulator with lateral p-i-n embedded photonic crystal nanocavity. Opt. Express 17, 22505-22513. https://doi.org/10.1364/OE.17.022505 (2009).

63. Chong, H. M. H. \& De La Rue, R. M. Tuning of photonic crystal waveguide microcavity by thermooptic effect. IEEE Photon. Technol. Lett. 16, 1528-1530 (2004).

64. Faraon, A. \& Vučković, J. Local temperature control of photonic crystal devices via micron-scale electrical heaters. Appl. Phys. Lett. 95, 043102. https://doi.org/10.1063/1.3189081 (2009).

65. Winfree, A. T. Biological rhythms and the behavior of populations of coupled oscillators. J. Theor. Biol. 16, 15-42. https://doi.org/ 10.1016/0022-5193(67)90051-3 (1967)

66. Haken, H. Basic concepts of synergetics. Appl. Phys. A 57, 111-115. https://doi.org/10.1007/BF00331431 (1993).

67. Yüce, E. et al. Adaptive control of necklace states in a photonic crystal waveguide. ACS Photon. 5, 3984-3988. https://doi.org/10. 1021/acsphotonics.8b01038 (2018).

68. Taguchi, Y., Takahashi, Y., Sato, Y., Asano, T. \& Noda, S. Statistical studies of photonic heterostructure nanocavities with an average q factor of three million. Opt. Express 19, 11916-11921. https://doi.org/10.1364/OE.19.011916 (2011).

69. Kogan, O., Rogers, J. L., Cross, M. C. \& Refael, G. Renormalization group approach to oscillator synchronization. Phys. Rev. E. 80, 036206. https://doi.org/10.1103/PhysRevE.80.036206 (2009).

70. Wächtler, C. W., Bastidas, V. M., Schaller, G. \& Munro, W. J. Dissipative nonequilibrium synchronization of topological edge states via self-oscillation. Phys. Rev. B. 102, 014309. https://doi.org/10.1103/PhysRevB.102.014309 (2020).

71. Rogister, F. \& Roy, R. Localized excitations in arrays of synchronized laser oscillators. Phys. Rev. Lett. 98, 104101. https://doi.org/ 10.1103/PhysRevLett.98.104101 (2007).

72. Vathakkattil Joseph, G. \& Pakrashi, V. Limits on anti-phase synchronization in oscillator networks. Sci. Rep. 10, 10178. https:// doi.org/10.1038/s41598-020-67021-6 (2020).

73. Scully, M. O. \& Lamb, W. E. Quantum theory of an optical maser. i. general theory. Phys. Rev. 159, 208-226. https://doi.org/10. 1103/PhysRev.159.208 (1967). 
74. Takemura, N., Takiguchi, M. \& Notomi, M. Low- and high- $\beta$ lasers in class-A limit: photon statistics, linewidth, and the laser-phase transition analogy. J. Opt. Soc. Am. B. 38, 699-710. https://doi.org/10.1364/JOSAB.413919 (2021)

75. Vinokur, V. M. et al. Superinsulator and quantum synchronization. Nature 452, 613-615. https://doi.org/10.1038/nature06837 (2008).

76. Flovik, V., Macià, F. \& Wahlström, E. Describing synchronization and topological excitations in arrays of magnetic spin torque oscillators through the kuramoto model. Sci. Rep. 6, 32528. https://doi.org/10.1038/srep32528 (2016).

\section{Author contributions}

N.T. developed the main theoretical idea and performed the simulations. K.T. taught us the injection locking of lasers and coupled-mode theory. K.T., M.T., and M.N. contributed to the discussion on photonic crystal laser technologies. N.T. drafted the manuscript.

\section{Competing interests}

The authors declare no competing interests.

\section{Additional information}

Supplementary Information The online version contains supplementary material available at https://doi.org/ 10.1038/s41598-021-86982-w.

Correspondence and requests for materials should be addressed to M.N.

Reprints and permissions information is available at www.nature.com/reprints.

Publisher's note Springer Nature remains neutral with regard to jurisdictional claims in published maps and institutional affiliations.

(c) (i) Open Access This article is licensed under a Creative Commons Attribution 4.0 International License, which permits use, sharing, adaptation, distribution and reproduction in any medium or format, as long as you give appropriate credit to the original author(s) and the source, provide a link to the Creative Commons licence, and indicate if changes were made. The images or other third party material in this article are included in the article's Creative Commons licence, unless indicated otherwise in a credit line to the material. If material is not included in the article's Creative Commons licence and your intended use is not permitted by statutory regulation or exceeds the permitted use, you will need to obtain permission directly from the copyright holder. To view a copy of this licence, visit http://creativecommons.org/licenses/by/4.0/.

(C) The Author(s) 2021 